\title{
Energy from streaming current and potential
}

\author{
Wouter Olthuis, Bob Schippers, Jan Eijkel and Albert van den Berg \\ $\mathrm{MESA}^{+}$Institute for Nanotechnology, University of Twente \\ P.O. Box 217, 7500 AE Enschede, The Netherlands
}

\begin{abstract}
Summary: It is investigated how much energy can be delivered by a streaming current source. A streaming current and subsequent streaming potential originate when double layer charge is transported by hydrodynamic flow. Theory and a network model of such a source is presented and initial experimental results are given, showing a supplied power of $20 \mathrm{nW}$ obtained by a pressure difference of $1 \mathrm{~atm}$ over a glass porous plug, using a 1-mM KCl solution. It is indicated how the rather low mechanical-to-electrical energy conversion efficiency can be increased.
\end{abstract}

Keywords: streaming potential, zeta potential, streaming current.

\section{Introduction}

Recently, a paper by Yang et al. [1] about energy from water attracted the attention of even the popular press. In that paper it was theoretically investigated how streaming current and potential from a water flow could be used to create electrical energy. In this paper we elaborate on this well-known concept [2] by giving experimentally obtained results, and indicate ways to optimize the supplied energy. When a solution is pressed through a porous plug (with pores in the micrometer or nanometer range, e.g. a sintered glass plug), a so-called streaming current is generated. This stems from the fact that generally the wall material is charged and a counter charge resides in the solution. When the mobile part of this counter charge is dragged along by the hydrodynamic flow, caused by the pressure difference over the porous plug, a net transport of charge occurs through the plug, the streaming current. Thus, charge polarization over the plug is created, resulting in an electric field, causing charge to move in the opposite direction of the streaming current. At a given hydrodynamic flow, equilibrium is reached: the net transport of charge through the porous plug is zero. The potential difference that is present over the plug in this zero-current situation is the (maximum) streaming potential. The streaming current can be treated as source, which can be connected to an external load. In this paper, some theoretical details of the origin and magnitude of the internal and external currents, as well as the streaming potential are given, resulting in an equivalent circuit. Regarding this circuit as a description of an energy source, its optimized energy transfer to an external load is discussed. The results of some initial experiments, focused on optimized transferred power, are presented and discussed.

\section{Theory}

The origin of the charge that forms the basis of the streaming potential stems from the chemical reactions taking place at the interface between de wall material of the porous plug and the aqueous solution flowing through it. Glass, for example, will dissociate protons at an intermediate solution $\mathrm{pH}$ according to $\mathrm{SiOH}<->$ $\mathrm{SiO}^{-}+\mathrm{H}^{+}$. This deprotonation results in an immobile negatively charged glass wall. This wall charge is compensated for by positive counter charge, present in the solution as ions. This arrangement of wall charge balanced by charges in the solution is referred to as the electrical double layer. A well-accepted description of this double layer is by the Gouy-Chapman-Stern model, basically subdividing the charge in the solution in two parts: an inner layer (the Stern layer) including immobile ions that are relatively strongly bound to the wall surface, and an outer, or diffuse layer, containing loosely bound mobile ions [3]. The distribution of charge in the diffuse layer causes an electrical potential with respect to the bulk of the solution, having a specific value, the zeta potential, $\zeta$, being the potential at the plane of shear. A pressure difference, $\Delta \mathrm{P}$, over the porous plug will cause a hydrodynamic flow dragging along the mobile charge past the shear plane. The electrical current thus created is the streaming current [4-6]:

$I_{s}=\frac{A \varepsilon_{0} \varepsilon_{r}}{\eta \ell} \Delta P \zeta$

with $\varepsilon_{0} \varepsilon_{\mathrm{r}}$ and $\eta$ the dielectric constant and viscosity of the solution, and $\ell$ and $\mathrm{A}$ the pore length and cross section of all pores, respectively. The electric field created by the resulting polarization of charge along the plug axis causes a force acting on the charges, resulting in a conduction current, $\mathrm{I}_{\mathrm{c}}$, in the opposite direction:

$I_{c}=A \sigma \frac{V_{s}}{\ell}$

with $\sigma$ the solution's conductivity and $\mathrm{V}_{\mathrm{s}}$ the voltage difference over the plug. Soon, a steady state is reached and the net transport of charge through the plug is zero: $I_{c}+I_{s}=0$. The potential difference, present over the plug in that case is the maximum streaming potential, $\mathrm{V}_{\mathrm{s}}$, from eqs. 1 and 2:

$V_{s}=\frac{\varepsilon_{0} \varepsilon_{r}}{\sigma \eta} \Delta P \zeta$

In order to investigate the energy supply of this source to an external load, $R_{\text {ext }}$, an equivalent circuit is presented, that fits the operational principle: a voltage controlled current source, shown in figure 1.

It is known form network theory that the maximum

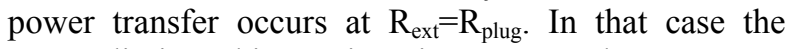
power dissipated in $\mathrm{R}_{\mathrm{ext}}$ is, using eqs. 1 and 3 [1]:

$$
P_{\text {ext, } \max }=\frac{1}{4} V_{s} \cdot I_{s}=\left(\frac{\Delta P \varepsilon_{0} \varepsilon_{r} \zeta}{\eta}\right)^{2} \frac{A}{4 \sigma \ell}
$$


In the rest of this paper, some experiments dedicated to the transfer of power are described and initial results are presented.

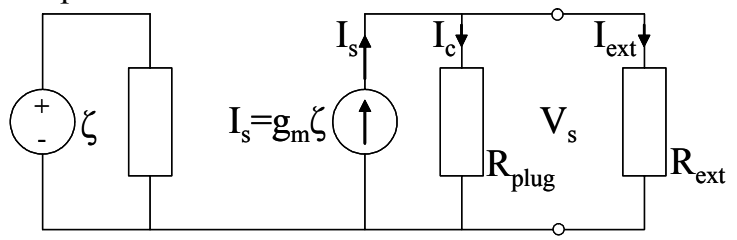

Fig. 1 Voltage contolled current source network model of the streaming current and potential.

\section{Experimental}

The set-up consisted of a 125-ml Duran Schott glass porous filter of porosity 5 , indicating a nominal pore size of $1.0-1.6 \mu \mathrm{m}$. The diameter of the filter was 60 $\mathrm{mm}$ and its thickness approx. $3.5 \mathrm{~mm}$. The outlet of the funnel was kept at $0.03 \mathrm{~atm}$ by a water jet pump. At both sides of the glass porous plug $\mathrm{Ag} / \mathrm{AgCl}$ electrodes were positioned to record the streaming potential with a PC-controlled HP34401A multimeter. It was independently determined that the charge transfer resistance of the $\mathrm{Ag} / \mathrm{AgCl}$ electrodes to the electrolyte solution was approx. $250 \Omega$, much lower than the porous plug resistance and therefore, this resistance could be neglected. The measurement set-up is schematically shown in figure 2.

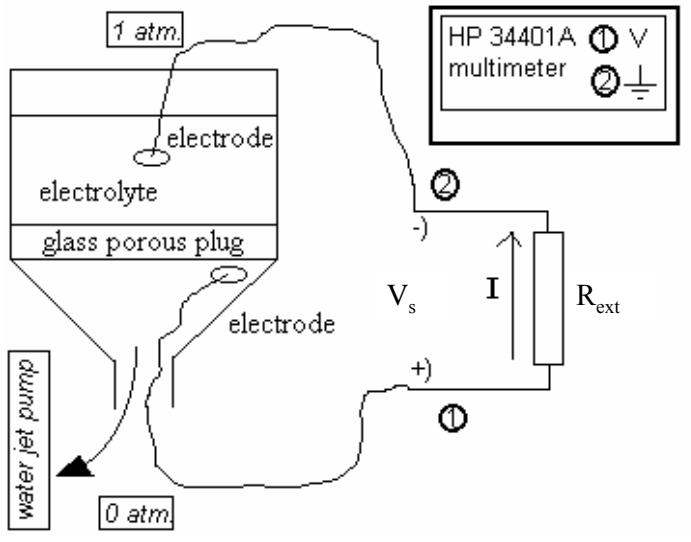

Fig. 2 The measurement set-up.

In order to increase the streaming potential, the $\mathrm{pH}$ of the electrolyte, sucked through the plug, was kept at $\mathrm{pH}$ 10 with $\mathrm{NaOH}$, thus increasing the zeta potential as explained in the previous section. The electrolyte consisted of either $1 \mathrm{mM}$ or $100 \mathrm{mM} \mathrm{KCl}$.

\section{Results}

First, the influence of the electrolyte conductivity, $\sigma$, was determined and the results are given in table 1 .

Table 1 Influence of [KCl] and $R_{e x t}$ on $P_{e x t}$.

\begin{tabular}{|l|l|l|l|l|}
\hline $\begin{array}{l}{[\mathrm{KCl}]} \\
(\mathrm{M})\end{array}$ & $\begin{array}{l}\mathrm{R}_{\text {ext }} \\
(\Omega)\end{array}$ & $\begin{array}{l}\mathrm{V}_{\mathrm{s}} \\
(\mathrm{V})\end{array}$ & $\begin{array}{l}\mathrm{I}_{\text {ext }} \\
(\mathrm{A})\end{array}$ & $\begin{array}{l}\mathrm{P}_{\text {ext }} \\
(\mathrm{W})\end{array}$ \\
\hline \multirow{2}{*}{$10^{-3}$} & $1 \cdot 10^{10}$ & $40 \cdot 10^{-3}$ & $\sim 0$ & $\sim 0$ \\
\cline { 2 - 5 } & $24 \cdot 10^{3}$ & $22 \cdot 10^{-3}$ & $9.2 \cdot 10^{-7}$ & $20 \cdot 10^{-9}$ \\
\hline \multirow{2}{*}{$10^{-1}$} & $1 \cdot 10^{10}$ & $1.2 \cdot 10^{-3}$ & $\sim 0$ & $\sim 0$ \\
\cline { 2 - 5 } & $1 \cdot 10^{4}$ & $7.5 \cdot 10^{-4}$ & $7.5 \cdot 10^{-8}$ & $5.6 \cdot 10^{-11}$ \\
\hline
\end{tabular}

In this table, both the open circuit streaming potential (very high $R_{e x t}$ ) is presented and the value of $R_{\text {ext }}$ where
$\mathrm{V}_{\mathrm{s}}$ drops to about half its maximum, indicating the value of $R_{\text {plug. }}$ In this situation, maximum power transfer to $\mathrm{R}_{\mathrm{ext}}$ is expected. In a qualitative sense, all expectations are met: a lower electrolyte concentration means a lower conductivity, $\sigma$, resulting in both a higher (maximum) $\mathrm{V}_{\mathrm{s}}$ and a higher supplied power to $R_{\text {ext }}$, as expected from eqs. 3 and 4, respectively. It is difficult to draw quantitative conclusions both from the fact that the nature and precise pore dimensions of the glass filter are not known and that the zeta potential itself is also, via the diffuse layer, a function of the electrolyte concentration. Finally, a series of measurements was performed with different loads, $R_{\text {ext }}$, using the lower concentration value, $1 \mathrm{mM} \mathrm{KCl}$. The results of both $\mathrm{V}_{\mathrm{s}}$ and $\mathrm{P}_{\mathrm{ext}}$ are presented in figure 3 .

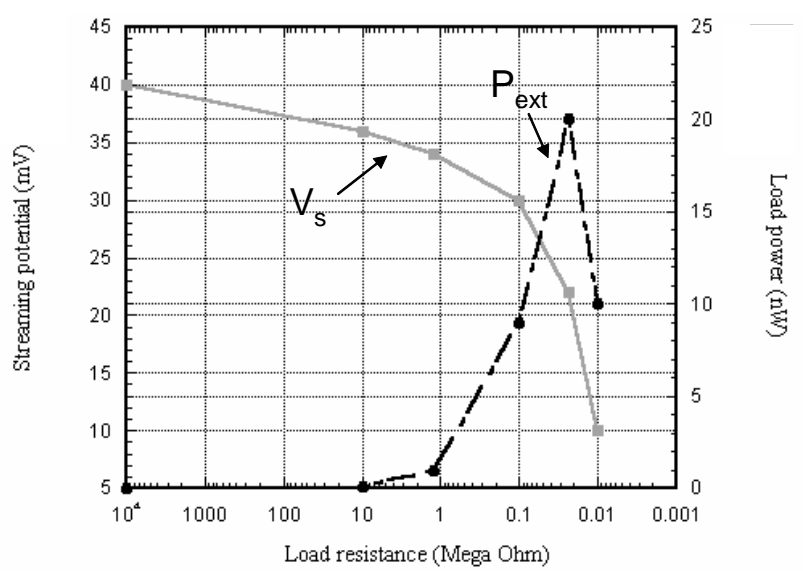

Fig. 3 Streaming potential $V_{s}$ and supplied power $P_{\text {ext }}$ as a function of $R_{\text {ext }}$ for $1 \mathrm{mM} \mathrm{KCl}$.

These curves nicely show the behaviour of the streaming source under load conditions, indicating a porous plug resistance of about $20 \mathrm{k} \Omega$, where $\mathrm{V}_{\mathrm{s}}=$ $1 / 2 V_{s, \max }$ and $\mathrm{P}_{\text {ext }}$ indeed obtains its maximum value (20 $\mathrm{nW}$ ) as expected from eq. 4. The energy conversion efficiency from the mechanical to the electrical domain is in this case far less than $1 \%$. A lower electrolyte concentration through a shorter plug will increase the efficiency.

\section{Final remarks}

It has been shown that energy can be obtained from a streaming potential source, according to the theoretical description. This description enables the proper design of a micromachined porous plug for optimized energy transfer, e.g., for Lab-on-a-Chip applications.

\section{References}

1. J. Yang, F. Lu, L.W. Kostiuk, D.Y. Kwok; J. Micromech. Microeng. 13 (2003), pp 963-970.

2. J.F. Osterle; J. Appl. Mech. 31 (1964) pp 161.

3. A.J. Bard, L.R. Faulkner; Electrochemical methods, Fundamentals and Applications, Wiley 1980.

4. P. Fievet, M. Sbaï, A. Szymczyk, A. Vidonne; J. Membr. Sci. 226 (2003), pp 227-236.

5. A. Yaroshchuk, V. Ribitsch; Langmuir 18 (2002), pp 20362038.

6. C. Werner, R. Zimmermann, T. Kratzmüller; Colloids and Surfaces 192 (2001), pp 205-213. 